\title{
An Editorial Note on Bioanalysis \& Biomedicine
}

\section{Sandeep Kumar Kar*}

Department of Cardiac Anesthesiology, Institute of Postgraduate Medical Education and Research, Kolkata, India

Journal of Bioanalysis \& Biomedicine (ISSN: 1948-593X) Hilaris publication, is an international prominent journal focusing on studies pertaining to Pharmaceutics, Pharmacology, Pharmacotherapy, Negative results of Biomedicine, Molecular Epidomology, Nanomedicine, Drug Development, Toxicology, Biomarkers is toxicology, Spectroscopic \& Chromatographic techniques etc.

It is an open access and peer reviewed journal by famous Editorial Board and the manuscripts are peer-reviewed by potential reviewers as indicated by their research interest.

Throughout the previous 3 years, published papers have been under the solid and able leadership of our Editor-in-Chief's Dr. Alexey Goltsov Particularly, we are grateful to for his persistent help and devotion towards the diary won't lost. We the distribution office of Journal of Bioanalysis and Biomedicine including me, we as a whole are respected and thankful for his benevolent dedication towards the journal.

We provide a fast pivot time workable for peer reviewing on and publishing the article online and to scatter the articles freely for exploration, educating and reference purposes.

The Journal of Bioanalysis and Biomedicine additionally fills in as an abridgment of information in pharmaco the study of disease transmission and drug safety which can be promptly gotten to by physicians, specialists, clinical researchers, general wellbeing experts, clinical understudies, clinicians, lab scientists, academicians and industry for keeping themselves refreshed and furthermore to detail further research programs.

The Journal of Bioanalysis and Biomedicine consistently distributes reports covering wide scope of subjects which incorporate new difficulties of over Pharmacology, Pharmacognosy and Phytochemistry just as different parts of biomedical sciences.

\section{Applied Strategies in 2019}

We have applied many strategies in 2019 , i.e.

- Using contacting through Mobile (Whatsapp) telephones

- Sent Call for papers for the famous in the field

- $\quad$ Approaching the Editors proposed prominent

- Approaching possible reviewers for the Call for papers

- Maintaining the frequency of articles.

\section{Conclusion}

I wish to give some point of view from the cycling research network of Journal of Bioanalysis and Biomedicine with respect to an ongoing Editorial note of our Journal.

I exclusively accept that the quality of the journal depends on the Eminence nearness in the Editorial Board just as Reviewer Board (possible reviewers) who endeavor to make the papers altogether productive and helpful for the scientific community.

How to cite this article: Sandeep Kumar Kar. "An Editorial Note on Bioanalysis \& Biomedicine." J Bioanal Biomed 12 (2020) doi: 10.37421/ JBABM.2020.12.234
*Address for Correspondence: Sandeep Kumar Kar, Department of Cardiac Anesthesiology, Institute of Postgraduate Medical Education and Research, Kolkata, India, E-mail: sndpkar@yahoo.co.in

Copyright: () 2020 Sandeep Kumar Kar. This is an open-access article distributed under the terms of the Creative Commons Attribution License, which permits unrestricted use, distribution, and reproduction in any medium, provided the original author and source are credited. 